\section{The Optimal Focusing Subspace for Coherent Signal Subspace Processing}

\author{
S. Valaee and P. Kabal
}

say, the center frequency of the spectrum of the signals, and transform the subspace at each frequency bin into the subspace created by the span of the location vectors at the focusing frequency. Then, they use a high-resolution algorithm such as MUSIC [8] to estimate the DOA's of the sources. They show that focusing reduces the resolution threshold sigual-to-noise ratio (SNR), which is defined as the SNR for a prescribed probability of resolution. They also show that if the integral of the signal covariance matrix taken over the frequency spectrum is full rank, the method can be applied to coherent signal localization. Hung and Kaveh [5] use a unitary variant of the CSM algorithm to avoid the focusing loss. They use the center frequency for focusing.

Swingler and Krolik [9] prove that for a single-source scenario, it is possible to have an unbiased estimate of the DOA's if the centroid of the source spectrum is selected as the focusing frequency. In [10], we showed that for multiple sources, the CSM algorithm cannot provide unbiased estimates of the DOA's. In this work, we propose a method to select the focusing subspace. The methed is based on minimizing a subspace fitting error. The subspace fitting error for each frequency bin is defined as the distance between the focusing matrix and the transformed location matrix. Later, we minimize a tight bound to the error. The simulation results show that using the method proposed here reduces the resolution threshold SNR and the bias of the DOA's estimates.

\section{THE CSM ALGORITHM}

Consider an array of $p$ sensors exposed to $q<p$ far-field wideband sources. The output of the sensors in the frequency domain is represented by

$$
\mathbf{x}(f)=\mathbf{A}(f, \theta) \mathbf{s}(f)+\mathbf{n}(f)
$$

where $s(f)$ and $n(f)$ are the Fourier transforms of the signal and noise vectors, and $\mathbf{A}(f, \theta)=\left[\mathbf{a}\left(f, \theta_{1}\right) \cdots \mathbf{a}\left(f, \theta_{g}\right)\right]$ is the full-rank $p \times q$ matrix of location vectors. It is assumed that the signal and noise samples are independent identicaily-distributed sequences of complex Gaussian random vectors- with unknown covariance matrices $\mathbf{S}(f)$ and $\sigma^{2} \mathbf{I}$, respectively. With these assumptions, the covariance matrix of the observation vector at the frequency $f_{j}$ is given by

$$
\mathbf{R}\left(f_{j}\right)=\mathbf{A}\left(f_{j}, \theta\right) \mathbf{S}\left(f_{j}\right) \mathbf{A}^{H}\left(f_{j}, \theta\right)+\sigma^{2} \mathbf{I}
$$

where the superscript $H$ represents the Hermitian transposition, For simplicity of notation, we suppress the frequency variable and represent $\mathbf{R}\left(f_{j}\right)$ by $\mathbf{R}_{j}, \mathbf{A}\left(f_{j}, \theta\right)$ by $\mathbf{A}_{j}$, and so on.

The CSM algorithm [11] is based on forming new observation vectors $\mathrm{y}_{j}$ such that

$$
\mathrm{y}_{j}=\mathbf{T}_{j} \mathbf{x}_{j}
$$

where the $\mathbf{T}_{j}$ 's are called the focusing matrices. In the unitary variant of the CSM algorithm [5], the $\mathbf{T}_{j}, j=1, \ldots, J$ are selected from

$$
\begin{aligned}
& \underset{\mathbf{T}_{j}}{\min }\left\|\mathbf{A}_{0}-\mathbf{T}_{j} \mathbf{A}_{j}\right\|, \\
& \text { subject to } \mathbf{T}_{j}^{H} \mathbf{T}_{j}=\mathbf{I}
\end{aligned}
$$

where $\|\cdot\|$ is the Frobenius matrix norm [4]. The solution to this minimization is given by [4], [5]

$$
\mathbf{T}_{j}=\mathbf{V}_{j} \mathbf{W}_{j}^{H}
$$

where $V_{j}$ and $W_{j}$ are the left and the right singular vectors of $\mathbf{A}_{0} \mathbf{A}_{j}^{H}$. In (5), $\mathbf{A}_{j}$ and $\mathbf{A}_{0}$ are assumed to be known. In practice, 
a preliminary step is required to determine these matrices. The preprocessing step involves using an ordinary beamformer to estimate the DOA's. These preestimated DOA's are then used to determine the focusing angles. The focusing angles are used in the structure of the location matrix to generate $\mathbf{A}_{j}$ and $\mathbf{A}_{0}$. An alternative method that avoids the pre-estimation step, but can only be applied to linear arrays, is spatial resampling, as proposed in [2] and [7] (see also [3])

The transformed observation vectors $y_{i}$ are used to form a sample correlation matrix for each frequency bin. An average of these aligned sample correlation matrices gives a universal sample correlation matrix that can be used for detection and DOA estimation. In the past, the focusing frequency in (5) has been chosen to be the center frequency of the spectrum of the signals. This choice is not optimal if the spectrum of the signal is asymmetric around the center frequency or the sampling in the frequency domain is nonuniform In the following section, we propose a method that finds the optimal focusing frequency.

\section{Focusing FreQuency SELEction}

Minimizing the subspace fitting error is an appropriate criterion for focusing frequency selection. In particular, the focusing frequency can be selected from

$$
\begin{aligned}
& \min _{f_{0}} \min _{\mathbf{T}_{j}} \sum_{j=1}^{J} w_{j}\left\|\mathbf{A}_{0}-\mathbf{T}_{j} \mathbf{A}_{j}\right\|^{2}, \\
& \text { subject to } \mathbf{T}_{j}^{H} \mathbf{T}_{j}=\mathbf{I},
\end{aligned}
$$

$$
\mathbf{A}_{\square} \in \mathcal{A}(\boldsymbol{\theta})
$$

where $w_{j}$ is a normalized weighting factor proportional to the SNR at the $j$ th frequency bin with $\sum_{j=1}^{J} w_{j}=1$, and $\mathcal{A}(\boldsymbol{\theta})$ is the set of all location matrices for the focusing angles $\theta$ and is defined by

$$
\mathcal{A}(\boldsymbol{\theta})=\{\mathbf{A}(f, \boldsymbol{\theta}) \mid f \in \mathcal{F}\}
$$

where $\mathcal{F}$ is an interval for the frequency $f$. The elements of this set are the location matrices with the DOA's $\theta$ and different frequencies. The following lemma holds for any location matrix.

Lemma 1: The location matrix $\mathbf{A}$ of an array with isotropic sensors located in an environment with uniform planar wavefronts satisfies

$$
\|\mathbf{A}\|^{2}=\sum_{i=1}^{q}\left\|\mathbf{a}_{i}\right\|^{2}=p q .
$$

Using Lemma 1 and (5), the subspace fitting error is given by

$$
\begin{aligned}
& \sum_{j=1}^{J} w_{j}\left\|\mathbf{A}_{0}-\mathbf{T}_{j} \mathbf{A}_{j}\right\|^{2} \\
& =\sum_{j=1}^{J} w_{j}\left[\left\|\mathbf{A}_{0}\right\|^{2}+\left\|\mathbf{A}_{j}\right\|^{2}-2 \Re \operatorname{tr}\left(\mathbf{A}_{0} \mathbf{A}_{j}^{H} \mathbf{T}_{j}^{H}\right)\right] \\
& =2 J p q-2 \sum_{j=1}^{J} \sum_{i=1}^{q} w_{j} \sigma_{i}\left(\mathbf{A}_{0} \mathbf{A}_{j}^{H}\right)
\end{aligned}
$$

where the $\sigma_{i}(\mathbf{B}), i=1, \ldots, q$ are the singular values of the matrix $\mathrm{B}$ arranged in nonincreasing order, $\Re(\cdot)$ represents the real part of a complex number, and $\operatorname{tr}(\cdot)$ is the trace operator. From (9), it is seen that the minimization problem (6) is identical to the maximization

$$
\begin{aligned}
& \max _{f_{0}} \sum_{j=1}^{J} \sum_{i=1}^{q} w_{j} \sigma_{i}\left(\mathbf{A}_{0} \mathbf{A}_{j}^{H}\right), \\
& \text { subject to } \mathbf{A}_{0} \in \mathcal{A}(\boldsymbol{\theta}) .
\end{aligned}
$$

Direct maximization of $(10)$ is tedious, and the complexity increases with the number of frequency samples. In the sequel, we

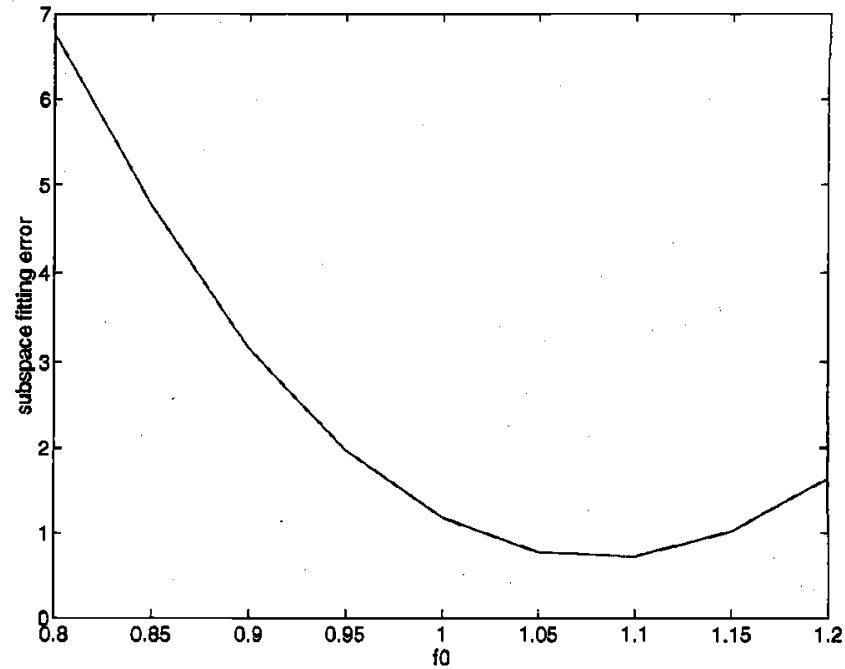

Fig. 1. Subspace fitting error (9) as a function of the focusing frequency for two uncorrelated far-field wideband sources at 10 and $14^{\circ}$ arriving at a linear array of eight sensors with a $20 \mathrm{~dB}$ SNR.

present a suboptimal method that is based on maximizing an upper bound to $(10)$.

The following lemma has been adopted from [4].

Lemma 2: If $\mathbf{A}, \mathbf{B} \in \mathbf{M}_{m, n}$ are given matrices with ordered singular values $\sigma_{1}(\mathbf{A}) \geq \cdots \geq \sigma_{q}(\mathbf{A}) \geq 0$ and $\sigma_{1}(\mathbf{B}) \geq \cdots \geq$ $\sigma_{q}(\mathbf{B}) \geq 0$, where $q=\min \{m, n\}$, then

$$
\|\mathbf{A}-\mathbf{B}\|^{2} \geq \sum_{i=1}^{q}\left[\sigma_{i}(\mathbf{A})-\sigma_{i}(\mathbf{B})\right]^{2} .
$$

Application of Lemma 2 to (9) results in the following lemma.

Lemma 3: For every $\mathbf{A}_{0}, \mathbf{A}_{j} \in M_{p, q}$ and $\sum_{i=1}^{q} w_{i}=1$

$$
\sum_{j=1}^{J} \sum_{i=1}^{q} w_{j} \sigma_{i}\left(\mathbf{A}_{0} \mathbf{A}_{j}^{H}\right) \leq \sum_{j=1}^{J} \sum_{i=1}^{q} w_{j} \sigma_{i}\left(\mathbf{A}_{0}\right) \sigma_{i}\left(\mathbf{A}_{j}^{H}\right) .
$$

The proposed method is based on maximizing the right-hand side of (12). Define

$$
\mu_{i} \triangleq \sum_{j=1}^{J} w_{j} \sigma_{i}\left(\mathbf{A}_{j}\right)
$$

The maximization problem is represented by

$$
\begin{aligned}
& \max _{f_{0}} \sum_{i=1}^{g} \mu_{i} \sigma_{i}\left(\mathbf{A}_{0}\right), \\
& \text { subject to } \mathbf{A}_{0} \in \mathcal{A}(\boldsymbol{\theta}) .
\end{aligned}
$$

This is a one-variable maximization problem that can be solved by searching for the best $f_{0}$. The computational complexity for the maximization (14) is independent of the number of frequency samples.

The simulation studies, which are reported in the following section, show that in the vicinity of the maximum point, the bound is tight. The tightness of the bound at the maximum point indicates that the method achieves a near-optimum subspace fit.

\section{Simulation RESUlTS}

Assume that a uniform linear array of eight sensors is exposed to two far-field wideband sources arriving from $10^{\circ}$ and $14^{\circ}$. The 


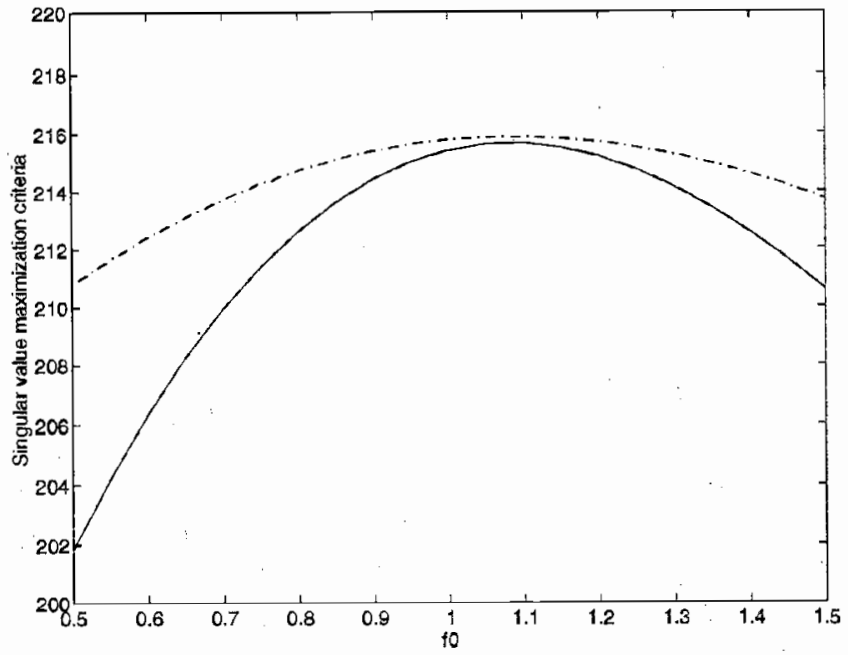

Fig. 2. Left- and right-hand-sides of (12) as functions of the focusing frequency for two uncorrelated far-field wideband sources at 10 and $14^{\circ}$ arriving at a linear array of eight sensors with a $20 \mathrm{~dB}$ SNR.

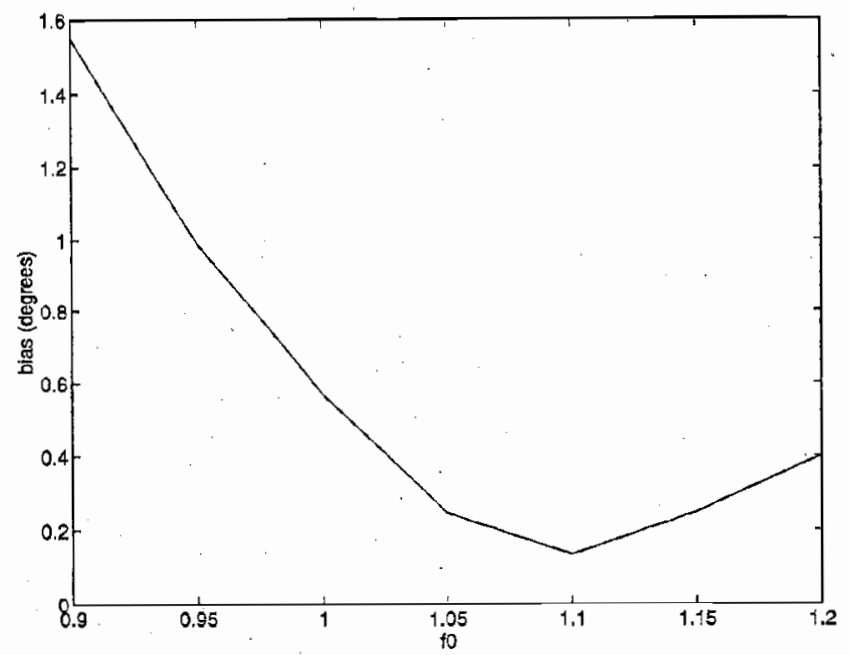

Fig. 3. Norm of the bias vector for the DOA estimation of two uncorrelated far-field wideband sources arriving at a linear array of eight sensors with a $20 \mathrm{~dB}$ SNR

signals are uncorrelated and have a $40 \%$ bandwidth relative to the center frequency. The spectrum for each of the signals is given by

$$
S(f)= \begin{cases}5 f-4 & 0.8 \leq f \leq 1.2 \\ 0 & \text { otherwise. }\end{cases}
$$

The spectrum is sampled using a 16-point FFT algorithm. The data at each frequency bin contain 100 snapshots. Using an ordinary beamformer, a single source is detected at $13^{\circ}$. We add two extra DOA's at $9^{\circ}$ and $17^{\circ}$ as the focusing angles. The subspace fitting error (9) is shown in Fig. 1. It is seen that the error is minimized at the frequency 1.1 , which is $10 \%$ higher than the center frequency and $3 \%$ higher than the centroid frequency. The left- and the righthand sides of (12) are compared in Fig. 2. Note that in the vicinity of the optimum point, the bound is tight. For a fixed SNR at $20 \mathrm{~dB}$, we have found the bias of the DOA estimates for different focusing frequencies. The results are depicted in Fig. 3. The bias of the DOA estimate for the centroid frequency is 0.1246 compared with 0.0487 for the optimum focusing frequency.
TABLE I

RESOLUTION OF THE CSM ALGORITHM FOR TWO CLOSELY SPACED Wimeband Stgnals as a Function of the Focusing Freolenct

\begin{tabular}{c|rrrr}
\hline & \multicolumn{4}{|c}{ SNR (dB) } \\
\cline { 2 - 5 }$f_{0}$ & -5 & 0 & 5 & 10 \\
\hline 0.90 & 0 & 6 & 36 & 64 \\
0.95 & 0 & 22 & 86 & 100 \\
1.00 & 2 & 41 & 99 & 100 \\
1.05 & 1 & 71 & 100 & 100 \\
1.10 & 6 & 89 & 100 & 100 \\
1.15 & 9 & 99 & 100 & 100 \\
1.20 & 11 & 100 & 100 & 100 \\
\hline
\end{tabular}

TABLE II

NORM OF THE BIAS VECTOR FOR TWO CIOSELY SPACED Wideband Signais as a Function of the Focusing Frequency

\begin{tabular}{c|cccc}
\hline & \multicolumn{4}{|c}{ SNR (dB) } \\
\cline { 2 - 5 }$f_{0}$ & -5 & 0 & 5 & 10 \\
\hline 0.90 & - & 1.22 & 1.48 & 1.55 \\
0.95 & - & 1.13 & 1.10 & 0.98 \\
1.00 & 1.57 & 0.88 & $\mathbf{0 . 6 8}$ & $\mathbf{0 . 5 7}$ \\
1.05 & 0.59 & 0.76 & 0.36 & 0.24 \\
1.10 & 1.08 & 0.54 & $\mathbf{0 . 2 2}$ & $\mathbf{0 . 1 3}$ \\
1.15 & 0.58 & 0.42 & 0.25 & 0.24 \\
1.20 & 0.79 & 0.37 & $\mathbf{0 . 3 9}$ & $\mathbf{0 . 4 0}$ \\
\hline
\end{tabular}

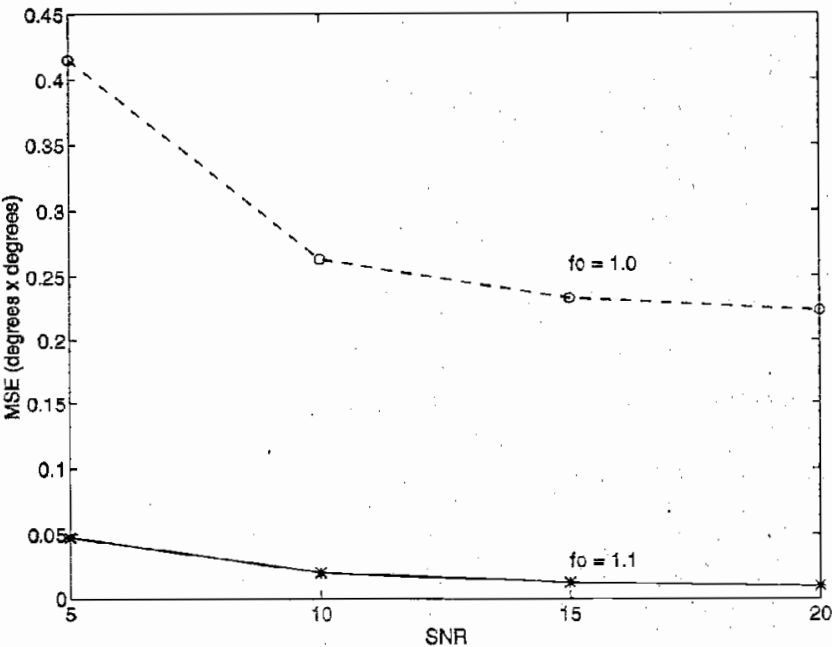

Fig. 4. Mean-square error of the CSM algorithm for the two focusing frequencies 1 and 1.1 and for two uncorrelated far-field wideband sources at $10^{\circ}$ and $14^{\circ}$ arriving at a linear array of eight sensors.

To compare the resolution capability of the CSM algorithm, we performed 100 independent trials and counted the number of times that the two DOA's were resolved as two peaks in the spectrum of the MUSIC algorithm. The results for different focusing frequencies are compared in Table I. The resolution threshold SNR at the optimum focusing frequency is lower than that for the center frequency. There are two factors that affect the resolution SNR. First, the optimum focusing frequency has a smaller subspace fitting error, and second, it is higher than the center frequency. The separation between the 


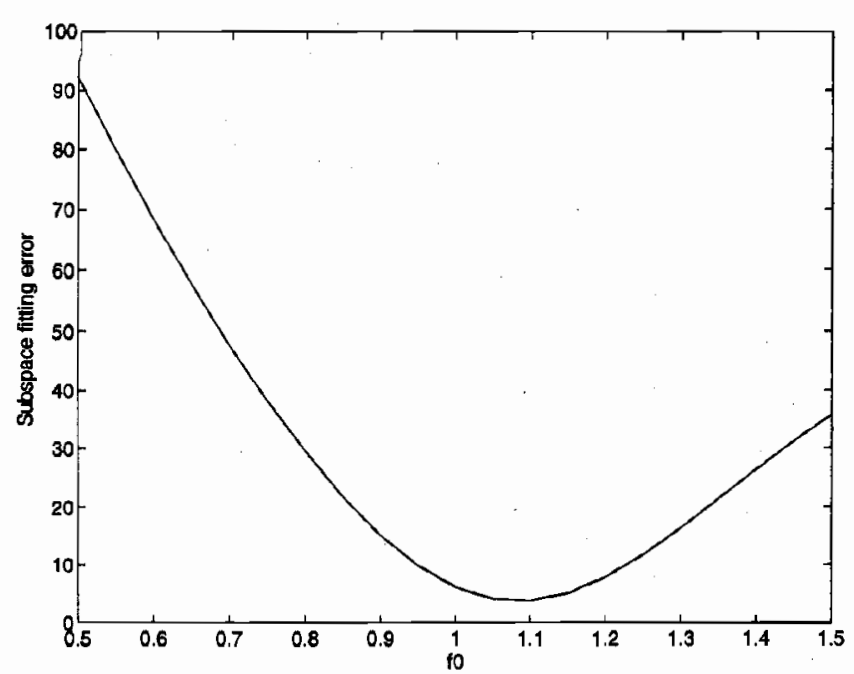

Fig. 5. Resolution of the CSM algorithm for two closely spaced wideband signals as a function of the focusing frequency.

TABLE III

Resolution of the CSM ALgORITHM FOR Four Wideband Signals as a Function of the Focusing Frequency

\begin{tabular}{c|rrrrrr}
\hline & \multicolumn{7}{|c}{ SNR (dB) } \\
\cline { 2 - 7 }$f_{0}$ & -5 & 0 & 5 & 10 & 15 & 20 \\
\hline 0.90 & 0 & 0 & 0 & 0 & 0 & 0 \\
0.95 & 0 & 0 & 0 & 0 & 0 & 0 \\
1.00 & 0 & 0 & 1 & 9 & 7 & 0 \\
1.05 & 0 & 0 & 19 & 76 & 99 & 100 \\
1.10 & 0 & 1 & 72 & 100 & 100 & 100 \\
1.15 & 0 & 9 & 92 & 100 & 100 & 100 \\
1.20 & 0 & 17 & 100 & 100 & 100 & 100 \\
\hline
\end{tabular}

location vectors for higher frequencies is larger, which results in a better resolution performance. The resolution at 1.2 is better than the other frequencies. However, the results of Table II and Fig. 3 show that for SNR's above the resolution threshold, the bias is minimum at the optimum focusing frequency. The mean-square error of the CSM algorithm for the two focusing frequencies 1.0 and 1.1 is compared in Fig. 4

We have also studied a multigroup scenario. In the second example, the signal of four sources located at $10^{\circ}, 14^{\circ}, 33^{\circ}$, and $37^{\circ}$ arrive at the same array as in the previous example. The focusing angles are $9^{\circ}, 13^{\circ}, 17^{\circ}, 31^{\circ}, 35^{\circ}$, and $39^{\circ}$. The spectrum of the signals are the same as the first example. The subspace fitting error (9) is depicted in Fig. 5. The optimum focusing frequency is again 1.1. Table III represents the resolution of the CSM algorithm for different SNR's and focusing frequencies. Note that at the center frequency 1.0 , detection is not possible even at high SNR's. If the number of sensors is increased, the array can detect signals using the center frequency for focusing. However, the resolution threshold for the optimum frequency is much smaller than that for the center frequency. The bias and the mean-square error are given in Tables IV and V. As these tables slow, the bias and the mean-square error for the optimum frequency are significantly smaller than the corresponding values for the center frequency. Thus, at $f_{0}=1.1$, we simultaneously get good resolution capability (Table III) and low bias (Table IV) over a range of SNR's.
TABLE IV

NORM OF THE BIAS VECTOR FOR FOUR WIDEBA.ND Signals as a Function of the Focusing Freqlency

\begin{tabular}{c|cccccc}
\hline & \multicolumn{7}{|c}{ SNR (dB) } \\
\cline { 2 - 7 }$f_{0}$ & -5 & 0 & 5 & 10 & 15 & 20 \\
\hline 0.90 & - & - & - & - & - & - \\
0.95 & - & - & - & - & - & - \\
1.00 & - & - & 2.05 & 1.66 & 1.51 & - \\
1.05 & - & - & 1.37 & 0.95 & 0.77 & 0.67 \\
1.10 & - & 1.74 & 0.90 & 0.44 & 0.28 & 0.20 \\
1.15 & - & 1.28 & 0.75 & 0.65 & 0.65 & 0.66 \\
1.20 & - & 1.35 & 0.96 & 1.02 & 1.02 & 1.04 \\
\hline
\end{tabular}

TABLE. V

NORM OF THE MEAN-SQUARE-ERROR VECTOR FOR FOUR Widebaid Signals as a Function of the Focusing Freqlency

\begin{tabular}{c|cccccc}
\hline & \multicolumn{7}{|c}{ SNR (dB) } \\
\cline { 2 - 7 }$f_{0}$ & -5 & 0 & 5 & 10 & 15 & 20 \\
\hline 0.90 & - & - & - & - & - & - \\
0.95 & - & - & - & - & - & - \\
1.00 & - & - & 2.78 & 1.67 & 1.27 & - \\
1.05 & - & - & 1.53 & 0.68 & 0.40 & 0.28 \\
1.10 & - & 2.11 & 0.64 & 0.16 & 0.06 & 0.03 \\
1.15 & - & 1.35 & 0.46 & 0.32 & 0.29 & 0.28 \\
1.20 & - & 1.49 & 0.73 & 0.71 & 0.67 & 0.68 \\
\hline
\end{tabular}

\section{SUMMARY}

In this correspondence, we have proposed a method to determine the optimal focusing frequency for the coherent signal-subspace method with unitary transformations. We have defined a criterion based on the subspace fitting error and optimized a tight upper bound to it. The simulation results show that the method successfully finds the global optimum value and improves the performance of the estimation by reducing the bias, the mean-square error, and the resolution SNR threshold.

\section{REFERENCES}

[1] K. M. Buckley and L. J. Griffiths, "Broad-band signal-subspace spatialspectrum (BASS-ALE) estimation," IEEE Trans. Acoust., Speech, Signal Processing, vol. 36. pp. 953-964, July 1988.

[2] H. Clergeot and O. Michel, "New simple implementation of the coherent signal subspace method for wideband direction of arrival estimation," in Proc. IEEE Int. Conf. Acoust., Speech, Signal Processing, Glasgow, Scotland, May 1989, pp. 2764-2767.

[3] W. Hong and A. H. Tewfik, "Focusing matrices for wideband array processing with no a priori angle estimates," in Proc. IEEE Int. Conf. Acoust., Speech, Signal Processing, San Francisco, CA, Mar. 1992, pp. II-493-II-496.

[4] R. A. Horn and C. A. Johnson, Matrix Analysis. Cambridge, UK: Cambridge University Press, 1985.

[5] H. Hung and M. Kaveh, "Focusing matrices for coherent signal-subspace processing." IEEE Trans. Acoust., Speech. Signal Processing, vol. 36. pp. 1272-1281, Aug. 1988.

[6] J. Krolik and D. N. Swingler, "Multiple broad-band source location using steered covariance matrices." IEEE Trans. Acoust., Speech, Signal Processing, vol, 37, pp. 1481-1494, Oct. 1989.

[7] _ "Focused wide-band array processing by spatial resampling," IEEE Trans. Acoust., Speech, Signal Processing, vol. 38, pp. 356-360, Feb. 1990. 
[8] R. O. Schmidt, "Multiple emitter location and signal parameter estimation," IEEE Trans. Antenna Propagat., vol. AP-34, pp. 276-280, Mar. 1986.

[9] D. N. Swingler and J. Krolik, "Source location bias in the coherently focused high-resolution broad-band beamformer," IEEE Trans. Acoust., Speech, Signal Processing, vol. 37, pp. 143-145, Jan. 1989.

[10] S. Valaee and P. Kabal, "Wideband array processing using a two-sided correlation transformation," IEEE Trans. Signal Processing, vol. 43, pp. 160-172, Jan. 1995.

[11] H. Hung and M. Kaveh, "Coherent signal-subspace processing for the detection and estimation of angles of arrival of multiple wide-band sources," IEEE Trans. Acoust, Speech, Signal Processing, vol. ASSP-33, pp. 823-831, Aug. 1985. 